\title{
Detection of Aspergillus fumigatus precipitins: a comparison of counter immunoelectrophoresis and double diffusion
}

\author{
D. W. WARNOCK \\ From the Department of Microbiology, Bristol Royal Infirmary, Bristol BS2 $8 \mathrm{HW}$
}

SUMMARY A counter immunoelectrophoresis method was compared with a double diffusion test for detecting Aspergillus fumigatus antibodies. Of 70 patients, 23 gave positive results in both tests and the remainder gave negative results. Fifteen patients with proven aspergillosis gave positive results in both tests.

The detection of Aspergillus fumigatus precipitins is of importance in the diagnosis of all forms of human aspergillosis. The principal serological method at present used to detect these antibodies is the double diffusion test. Counter immunoelectrophoresis (CIEP) is a simple and rapid method which has been reported to be useful for detecting antibodies in persons with fungal infections including candidiasis, coccidioidomycosis, and histoplasmosis (Gordon et al., 1971; Hellwege et al., 1972; Kleger and Kaufman, 1973; Odds et al., 1975). Previous reports (Ward and Kohler, 1973; Dee, 1975) have indicated that CIEP compares well with double diffusion for the detection of $A$. fumigatus precipitins in patients with aspergillomata. The CIEP method has not, however, been compared with double diffusion for precipitin detection in patients with asthma or allergic aspergillosis. The purpose of this investigation was to determine the usefulness of CIEP in patients with different forms of aspergillosis. The findings are compared with those for the double diffusion test.

\section{Methods}

\section{SUBJECTS}

Serum was obtained from 15 patients with proven $A$. fumigatus infection (five patients with aspergillomata; 10 patients with allergic aspergillosis), from 50 persons with asthma, and from five persons with bronchiectasis or fibrosis. The clinical and radiographic features used in the selection of the patients with aspergillosis have been described in detail elsewhere (Golberg, 1962; Henderson, 1968).

Received for publication 20 September 1976
DOUBLE DIFFUSION TEST

Two commercial $A$. fumigatus antigens (Bencard) were used at concentrations of $20 \mathrm{mg}$ per $\mathrm{ml}$. The medium for this test was prepared by dissolving Noble agar (Difco) at 3\% concentration in 0.9\% saline, cooling to $60^{\circ} \mathrm{C}$, and then adding an equal

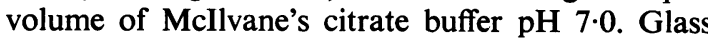
slides were coated to a depth of $1.5 \mathrm{~mm}$ and a template was used to cut a pattern of peripheral antigen wells of $4 \mathrm{~mm}$ diameter arranged $6 \mathrm{~mm}$ (edge to edge) from a $12.5 \mathrm{~mm}$ diameter central serum well. Diffusion was carried out in moist chambers for up to one week at room temperature. The slides were rinsed in $\mathbf{0 . 1} \mathrm{M}$ saline overnight, dried, and stained with $0.5 \%$ Coomassie Brilliant Blue R.

\section{COUNTER IMMUNOELECTROPHORESIS (CIEP)}

Glass slides were coated to a depth of $1.5 \mathrm{~mm}$ with $1 \%$ agarose (Meath) dissolved in $0.05 \mathrm{M}$ veronal buffer pH $8 \cdot 2$. Wells of $4 \mathrm{~mm}$ diameter, set $4 \mathrm{~mm}$ apart, were cut in the gel; serum specimens were placed in the anodic wells. Electrophoresis was carried out with $0.05 \mathrm{M}$ veronal buffer $\mathrm{pH} 8.2$ as the reservoir buffer. A constant current of $10 \mathrm{~mA}$ was applied for 2 hours at room temperature. The slides were rinsed in $5 \%$ tri-sodium citrate for 15 minutes, then in $0.1 \mathrm{M}$ saline overnight, dried, and stained with $0.5 \%$ Coomassie Brilliant Blue $\mathbf{R}$.

A range of $A$. fumigatus antigen (Bencard) dilutions was first tested against a number of positive serum specimens from persons with aspergillosis to minimise the incidence of false-negative results due to antigen excess or precipitation at or in the anodic (serum) well. A concentration of $5 \mathrm{mg}$ per $\mathrm{ml}$ was found to give optimum results for both antigens with 
regard to the position and definition of the precipitin lines produced.

\section{Results}

The table summarises the results of $A$. fumigatus precipitin detection in the different groups of patients using the CIEP method and the double diffusion test. Altogether 23 persons, including the 15 patients with aspergillosis, gave positive results in both tests. The remaining persons gave negative results in both tests.

Of the 23 persons with precipitins, those with aspergillomata gave multiple precipitin lines in both tests. In contrast, the patients with asthma or allergic aspergillosis gave single or double lines in both tests.

Table Comparison of double diffusion (DD) and CIEP for the detection of $\mathrm{A}$. fumigatus precipitins

\begin{tabular}{llcc}
\hline Diagnosis & $\begin{array}{l}\text { Number } \\
\text { of persons } \\
\text { tested }\end{array}$ & $\begin{array}{l}\text { Number of persons giving } \\
\text { positive results: }\end{array}$ \\
\cline { 3 - 4 } & & DD test & CIEP test \\
\hline Aspergilloma & 5 & 5 & 5 \\
Allergic aspergillosis & 10 & 10 & 10 \\
Asthma & 50 & 6 & 6 \\
Bronchiectasis or fibrosis & 5 & 2 & 2 \\
\hline
\end{tabular}

\section{Discussion}

The findings of this investigation confirm previous reports (Ward and Kohler, 1973; Dee, 1975) that CIEP can be used for the rapid detection of $A$. fumigatus precipitins in patients with aspergillomata. The results indicate that this method compares well with double diffusion for precipitin detection in persons with asthma or allergic aspergillosis. The number of precipitin lines observed in both tests appeared to be related to the clinical form of $A$. fumigatus infection.

Other investigations have revealed that both the double diffusion test and the CIEP method for detecting $A$. fumigatus precipitins are specific: antibodies have not been detected in persons with other fungal or bacterial infections, or in normal persons (Coleman and Kaufman, 1972; Dee, 1975). Precipitins have, however, been detected in some asthmatic patients without definite aspergillosis and in patients with bronchiectasis or fibrosis (Longbottom and Pepys, 1964; Henderson et al., 1968). Their presence has been attributed to occult infection with $A$. fumigatus.

In conclusion the results indicate that CIEP is a sensitive method for detecting $A$. fumigatus precipitins and suggest that it has potential diagnostic applications in aspergillosis.

\section{References}

Coleman, R. M. and Kaufman, L. (1972). Use of the immunodiffusion test in the serodiagnosis of aspergillosis. Appl. Microbiol., 23, 301-308.

Dee, T. H. (1975). Detection of Aspergillus fumigatus serum precipitins by counter immunoelectrophoresis. J. clin. Microbiol., 2, 482-485.

Golberg, B. (1962). Radiological appearances in pulmonary aspergillosis. Clin. Radiol., 13, 106-114.

Gordon, M. A., Almy, R. E., Greene, C. H., and Fenton, J. W. (1971). Diagnostic mycoserology by immunoelectroosmophoresis. Amer. J. clin. Path., 56, 471-474.

Hellwege, H. H., Fischer, K., and Bläker, F. (1972). Diagnostic value of Candida precipitins (Letter). Lancet, 2, 386.

Henderson, A. H. (1968). Allergic aspergillosis: review of 32 cases. Thorax, 23, 501-512.

Henderson, A. H., English, M. P., and Vecht, R. J. (1968). Pulmonary aspergillosis. Thorax, 23, 513-518.

Kleger, B. and Kaufman, L. (1973). Detection and identification of diagnostic Histoplasma capsulatum precipitates by counterelectrophoresis. Appl. Microbiol., 26, 231-238.

Longbottom, Joan L. and Pepys, J. (1964). Pulmonary aspergillosis: diagnostic and immunological significance of antigens and C-substance in Aspergillus fumigatus. J. Path. Bact., 88, 141-151.

Odds, F. C., Evans, E. G. V., and Holland, K. T. (1975). Detection of Candida precipitins: a comparison of double diffusion and counter immunoelectrophoresis. J. immunol. Methods, 7, 211-218.

Ward, G. W., Jr. and Kohler, P. F. (1973). Counterelectrophoresis as a rapid method for the detection of Aspergillus precipitins in pulmonary disease. Chest, 63, Supplement, 49-51. 